\title{
Movimentos oculares e padrões de busca visual em tarefas de rotação mental
}

\author{
Priscila Coure \\ Elizen Coutinho de Macedo ${ }^{1}$ \\ Fernando César Capovilla \\ José Salomão Schwartzman
}

\section{Resumo}

O objetivo deste estudo é identificar as estratégias utilizadas nas tarefas de rotação mental pela análise dos traçados dos movimentos oculares. Foi analisado o desempenho de 40 participantes na comparação de pares de objetos tridimensionais rotacionados no eixo y, com diferenças de angulação de $0^{\circ}$ a $180^{\circ}$. Foi utilizado um sistema computacional de rastreamento dos movimentos oculares (eyetracking) durante a visualização de figuras. Os resultados mostram que tempo de julgamento, duração média das fixações do olho, número de fixações nos objetos e número de alternâncias entre os dois objetos aumentam em função da diferença de angulação. Análise dos traçados oculares, com base na inspeção visual, indica o uso de dois tipos de estratégias: rotação mental em torno dos eixos padrões e comparação independente da orientação. O uso dessas estratégias é discutido com relação ao desempenho dos participantes e à memória de trabalho.

Palavras-Chave: Rotação mental; Busca visual; Direção do olhar; Eyetracking.
\end{abstract}

\section{Eye movements and scan patterns in mental rotation tasks}

\begin{abstract}
The purpose of this paper is to identify the strategies used on mental rotation tasks by analyzing ocular movements. The performance of 40 participants on comparison of pairs of three-dimensional objects, rotated on the y-axis, from $0^{\circ}$ to $180^{\circ}$ was analyzed. An eye-tracking computerized system was used to track eye movements during scene visualization. Results showed that judgment time, fixation duration, number of fixations and number of switches between the objects increased with rotation angle. Analysis by visual inspection indicates the use of two kinds of strategies: Mental rotation around the standard axis and comparison of orientation-free descriptions. The use of the strategies is discussed with regard to performance and working memory.

Keywords: Mental rotation; Scan pattern; Eyegaze; Eyetracking.
\end{abstract}

\section{Introdução}

O estudo dos movimentos oculares tem contribuído para a compreensão dos processos cognitivos ao longo dos últimos 20 anos (Rayner, 1998). Leitura e percepção de cenas são os focos principais desses estudos, mas não os únicos. Com base na análise da direção e fixação dos olhos também se pode inferir a respeito da estratégia cognitiva utilizada em tarefas como a rotação mental (Just \& Carpenter, 1976).

A rotação mental é uma função cognitiva complexa que se baseia na hipótese de equivalência funcional (Sternberg, 2000), que foi primeiramente demonstrada no trabalho de Shepard e Metzler (1971). Naquele estudo solicitava-se aos participantes que observassem pares de figuras bidimensionais mostrando formas geométricas tridimensionais. Essas formas sofriam rotação de zero a 180 graus, no plano da figura ou em profundidade. Eram apresentadas, também, figuras distraidoras que não eram rotações dos estímulos originais. Os participantes deviam compará-las e determinar se as duas figuras eram congruentes com relação à forma tridimensional, independentemente da orientação, ou se eram objetos diferentes. Os resultados mostraram que os tempos de reação formam uma função linear da diferença angular entre os dois objetos tridimensionais, ou seja, para cada aumento no grau de rotação das figuras, houve um acréscimo correspondente nos tempos de reação.

Tais resultados são funcionalmente equivalentes aos que se poderiam esperar se as pessoas estivessem fazendo a rotação de objetos físicos no espaço. Ou seja, demora-se mais para fazer a rotação de objetos em maiores ângulos de rotação, não importando se giram no sentido horário, anti-horário ou na terceira dimensão da profundidade (Sternberg, 2000).

A rotação mental tem sido uma das funções cognitivas mais estudadas, e os achados confirmam a idéia de que seja análoga à rotação física (Desrocher,

Apoio: Mackpesquisa, CNPq, CAPES

${ }^{1}$ Endereço para correspondência:

E-mail: ecmacedo@mackenzie.com.br 
Smith \& Taylor, 1995). Estudos realizados a partir da década de 1980 mostraram que a rotação mental estaria relacionada com o planejamento para ação e com ativação de áreas cerebrais específicas.

Usando fotos de mãos humanas, Sekyiama (1982, 1983, apud Fischer, Deubel, Wohlschlager \& Schneider, 1999) demonstrou que a tarefa de rotação mental pode ser resolvida pela imaginação do movimento da própria mão, com indícios de uma ligação entre a tarefa e o planejamento da ação. Recentemente, foi demonstrado que isso também é verdadeiro com as figuras tridimensionais de Shepard e Metzler (Wohlschlager \& Wohlschlager, 1998).

Seres humanos podem adotar estratégias motoras na rotação mental com objetos que não sejam necessariamente partes do corpo (Wraga, Thompson, Alpert \& Kosslyn, 2003). As evidências de que isso ocorre podem ser percebidas em tarefas simultâneas de rotação motora compatíveis com as de rotação mental, que resultam na redução de erros e de tempo de reação. Ao contrário, tarefas simultâneas de rotação motora conflitante com a mental causam interferência, aumentando significativamente o tempo de resposta e o número de erros, conforme demonstrado em diversos estudos (Wexler, Kosslyn \& Berthoz, 1998; Wohlschlager \& Wohlschlager, 1998; Wraga et al., 2003). Corroborando esses achados, estudos de neuroimagem têm mostrado que estruturas motoras são ativadas não apenas durante um movimento motor mas, também, em tarefas que não requerem movimento motor, como na rotação mental (Ganis, Keenan, Kosslyn \& Pascual-Leone, 2000).

Shepard e Metzler (1971) propuseram que, ao resolver o problema, os participantes rotacionassem mentalmente um dos estímulos em congruência com o outro para decidir se eram diferentes ou iguais. Desrocher et al. (1995) apontam para a divisão desta tarefa em três estágios. O primeiro estágio é o de busca ou avaliação, que serve para codificar figuras inteiras ou identificar partes que sejam semelhantes nos dois estímulos. $\mathrm{O}$ segundo estágio pode ser compreendido de duas maneiras, envolvendo a rotação mental da figura inteira ou de parte dela até que sua orientação esteja similar à do modelo. O terceiro estágio envolve confirmação da coerência da transformação e a tomada de decisão sobre se as figuras são diferentes ou iguais. Estudos indicam equivalência estratégica entre homens e mulheres, e que os homens são superiores apenas em relação à rapidez (Sanders, Soares \& D'Aquila, 1982).

Algumas questões, no entanto, não poderiam ser respondidas com base somente na análise do tempo de reação. A rotação da figura é feita por inteiro ou apenas parte dela é rotacionada? Como o participante sabe que partes da figura devem ser rotacionadas, ou seja, quais partes das figuras são possivelmente correspondentes entre si? Como o participante sabe o quanto rotacionar um objeto? Neste caso, poder-se-ia supor uma rotação balística, na qual o indivíduo faz uma estimativa da disparidade ou um processo de rotação monitorado em diversos pontos?

Segundo Just e Carpenter (1976), os padrões e as durações das fixações dos olhos durante uma tarefa que envolva input visual refletem as operações mentais que ocorrem na memória de trabalho. Desta forma, é possível que a localização, duração e seqüência das fixações numa tarefa de rotação mental indiquem como a informação visual é manipulada internamente.

Quando olhamos uma cena, lemos ou procuramos por algum objeto, fazemos os chamados movimentos oculares sacádicos. Entre um movimento sacádico e outro, os olhos permanecem parados durante as fixações. É provavelmente nessas pausas que programamos a orientação e a direção do próximo movimento sacádico, o que indica a presença de atenção e processamento cognitivo (Rayner, 1998).

Usando equipamentos de rastreamento dos movimentos oculares para estudar a rotação mental, Just e Carpenter $(1976,1985)$ notaram que os participantes olhavam sistematicamente para um e outro objeto. Encontraram efeito da angulação na alternância entre os objetos do lado esquerdo e do direito. A cada aumento de $40^{\circ}$ na angulação das figuras, houve acréscimo de aproximadamente uma alternância. Tais dados sugerem que as rotações não sejam balísticas, mas monitoradas a cada passo, possivelmente a cada 45 graus.

A divisão da tarefa em estágios foi corroborada no experimento de Just e Carpenter (1976). Aparentemente, a memória de trabalho não opera com a figura inteira, mas com um segmento dela a cada passo. No estágio inicial da busca, o participante identifica o segmento de um objeto e procura pelo segmento correspondente no outro objeto. $\mathrm{Na}$ transformação e comparação, as fixações se concentram nos segmentos escolhidos de um e outro objeto repetidamente. $\mathrm{Na}$ confirmação, o participante determina se outros segmentos também são correspondentes quando rotacionados.

Segundo Just e Carpenter (1985), há quatro tipos de estratégia em tarefas de rotação mental: 1) rotação mental em torno de eixos padrões; 2) rotação mental em torno de eixos definidos pela tarefa; 3) comparação independente da orientação; e 4) mudança de perspectivas. Todas elas foram deduzidas a partir do relato verbal dos participantes ao realizarem a tarefa.

A estratégia de rotação mental em torno de eixos padrões ( $x, y$ e $z$ ) é a mais comumente descrita pela literatura psicológica. Nela, o participante rotaciona mentalmente o objeto em um dos eixos padrões.

Psico-USF, v. 10, n. 1, p. 41-49, jan./jun. 2005 
A estratégia de rotação mental em torno de eixos definidos pela tarefa pode ocorrer em atividades como a comparação de cubos rotacionados. Em vez de usar os eixos usuais para a rotação do cubo, o participante faz a rotação por um eixo arbitrário, como o diagonal formado pelos cantos do cubo.

$\mathrm{Na}$ estratégia de comparação independente da orientação, o participante se baseia nas relações espaciais de uma figura e as compara com aquelas presentes em outra para verificar se as mesmas relações são mantidas. Nesse caso, não realiza transformação espacial.

Por fim, na estratégia de mudança de perspectivas, a posição do objeto e do participante em relação a ele são codificadas como um sistema de coordenadas. Para a resolução do problema, o participante se imagina em outra posição com relação ao objeto, o que possibilitaria vê-lo em outra orientação.

O presente estudo buscou avaliar os padrões de busca visual em tarefas de rotação mental por participantes não treinados para a tarefa, com o objetivo de identificar a existência de padrões, explorar as estratégias usadas e descrever a relação entre o tipo de padrão utilizado e o desempenho do participante.

\section{Método}

\section{Participantes}

Quarenta estudantes do curso de psicologia, vinte homens ( $\mathrm{M}=24,1 ; \mathrm{DP}=3,86$ anos de idade) $\mathrm{e}$ vinte mulheres ( $M=23,35$; $D P=2,23$ anos de idade).

\section{Equipamento}

Para a apresentação dos estímulos e rastreamento dos movimentos oculares foi usado o Sistema Eyegaze ${ }^{\circledR}$ desenvolvido pela LC Technologies Inc. (2001). Este sistema registra os movimentos oculares do participante automaticamente e em tempo real. A direção do olhar é determinada pelo método de reflexo da córnea no centro da pupila (PCCR-Pupil-Center-Corneal Reflexion). O equipamento é calibrado com apenas um dos olhos, e as medidas são feitas em uma taxa de amostragem de 60 Hertz. Para cada amostragem, são geradas as seguintes informações: flag indicando se a imagem do olho foi capturada pela câmera; diâmetro e posição da pupila a partir das coordenadas x e y do plano da tela do computador; deslocamento do globo ocular ao longo do eixo $z$; duração e localização das fixações e movimentos sacádicos.

Foi usado um microcomputador com processador Intel Pentium ${ }^{\circledR}$ III, $128 \mathrm{Mb}$ de memória RAM, placa de vídeo de $2 \mathrm{Mb}$, monitor de vídeo com tela plana do tipo Liquid Crystal Display de 15" com resolução de 800 x 600 pixels, suporte para monitor de câmera com lentes sensíveis a infravermelho de alta velocidade (RS170 ou CCIR) e Light Emitting Diode (LED) emissor de raios infravermelhos.

\section{Estímulos}

Foram reproduzidos e ampliados três objetos tridimensionais dentre os cinco utilizados no trabalho de Capovilla, Guedes e Macedo (1998), com base nos criados por Shepard e Metzler (1971). Todos os estímulos eram compostos por 10 blocos, arranjados espacialmente para formar diferentes objetos tridimensionais.

Os estímulos foram rotacionados nos ângulos $0^{\circ}, 45^{\circ}, 90^{\circ}, 135^{\circ}$ e $180^{\circ}$ apenas no eixo y, pois, segundo dados de Capovilla et al. (1998), o tempo de julgamento dos objetos congruentes (identidade) é menor nesse eixo. Cada um dos três objetos (A, B, C) foi rotacionado 5 vezes, nos ângulos $0^{\circ}, 45^{\circ}, 90^{\circ}, 135^{\circ}$ e $180^{\circ}$, gerando quinze figuras, sendo 5 referentes a cada objeto. As cinco figuras referentes ao objeto A (A1, A2, A3, A4 e A5) foram tomadas como modelo de comparação. Já as figuras B1 a B5 e C1 a C5 foram tomadas como distraidoras. Foi analisado o desempenho dos participantes apenas nas comparações entre as figuras derivadas do objeto A (A1 a A5). Enquanto aquelas entre objetos diferentes (A e $\mathrm{B}$ ou $\mathrm{A}$ e $\mathrm{C}$ ) foram ignoradas, tendo sido as figuras $\mathrm{B}$ e $\mathrm{C}$ usadas apenas como distraidoras, para contrabalançar os julgamentos de diferença.

Cada objeto (A, B e C) foi comparado com ele mesmo cinco vezes. No total, foram apresentados 30 quadros para os participantes, dos quais 15 representavam os objetos intrinsecamente congruentes (identidade) e os outros 15, incongruentes (diferença). A Figura 1 ilustra todas as figuras referentes ao objeto A usado como referência para as rotações (A1 a A5) e um exemplo dos objetos distraidores (B1 e C1). 


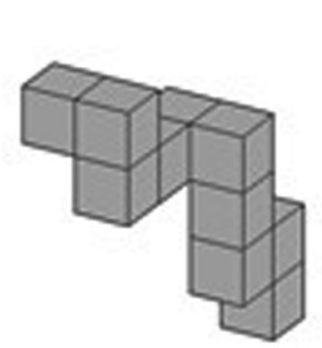

A 1

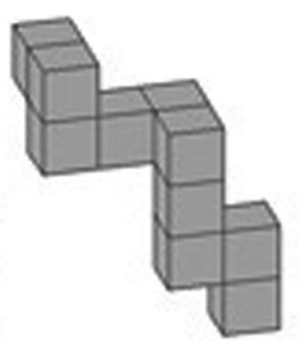

A 2

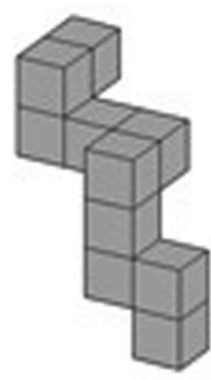

A 3
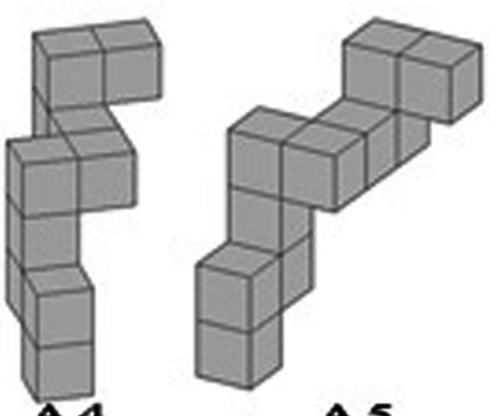

A5
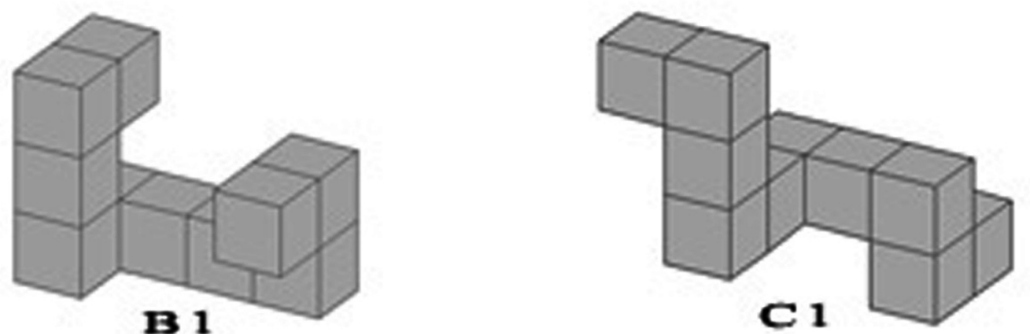

Figura 1 - Objetos tridimensionais usados como referência para rotação

Cada quadro de apresentação tinha $800 \times 600$ pixels, ocupando toda a região da tela do computador e possuía dois objetos a serem comparados, além dos sinais de igual e diferente na parte superior do quadro, para onde o participante deveria olhar conforme seu julgamento.

\section{Procedimento}

Cada sessão foi individual, com a duração média de 15 minutos. As instruções foram dadas por escrito, em virtude da complexidade da tarefa e da necessidade de exemplos para melhor compreensão. Após a leitura das instruções, os participantes apoiavam a cabeça no suporte de imobilização e o equipamento era calibrado para um dos olhos do participante. $\mathrm{O}$ experimento era iniciado sem a realização de treino, a fim de avaliar o comportamento das pessoas em uma situação de novidade da tarefa.

Nas tarefas de rotação mental, o participante devia comparar duas figuras que representam objetos tridimensionais e inferir se as figuras representam o mesmo objeto (identidade) ou objetos diferentes (diferença). Foi dito ao participante que duas figuras representam o mesmo objeto quando a rotação de uma delas resulta no outro, e objetos diferentes quando a rotação é incapaz de fazê-lo. Foi explicado que ele devia julgar se as figuras representavam ou não o mesmo objeto. Então, olhar para o sinal "igual" no primeiro caso, e para o sinal "diferente" no segundo. Assim que tivesse emitido a resposta, devia avisar o examinador. Foram apresentados 30 quadros, em ordem aleatória. A Figura 2 ilustra um dos quadros apresentados ao participante, com o objeto A rotacionado a $90^{\circ}$ no eixo y e os sinais de "igual" e "diferente". Neste caso, o participante devia olhar para o sinal de "igual".
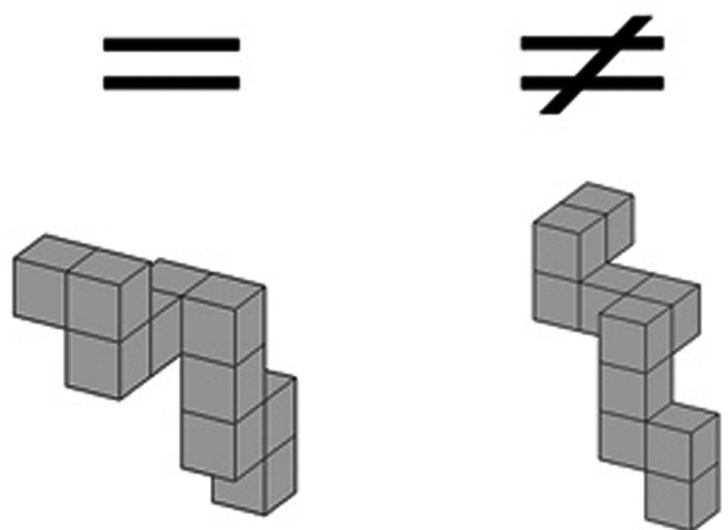

Figura 2 - Quadro com o objeto A (esquerda) e o mesmo objeto rotacionado a $90^{\circ}$ (direita)

Para avaliar o efeito da angulação sobre as medidas obtidas, foram conduzidas análises de variância unifatoriais usando o programa de análise estatística SPSS 11.5. A variável independente foi o grau de angulação com cinco níveis: $0^{\circ}, 45^{\circ}, 90^{\circ}, 135^{\circ}$ e $180^{\circ}$. As variáveis dependentes foram: número de respostas corretas; tempo de julgamento, número total de fixação nos objetos; duração total das fixações nos objetos e número de alternâncias entre os dois objetos.

Psico-USF, v. 10, n. 1, p. 41-49, jan./jun. 2005 


\section{Resultados}

O Tempo de Julgamento (TJ) foi obtido a partir da identificação do momento da primeira fixação na região de resposta, desde que o participante a tivesse confirmado verbalmente. A Figura 2 mostra que o TJ foi função linear crescente da diferença de angulação entre as figuras do mesmo objeto $\mathrm{A}$ na faixa de $0^{\circ}$ a $180^{\circ}$.

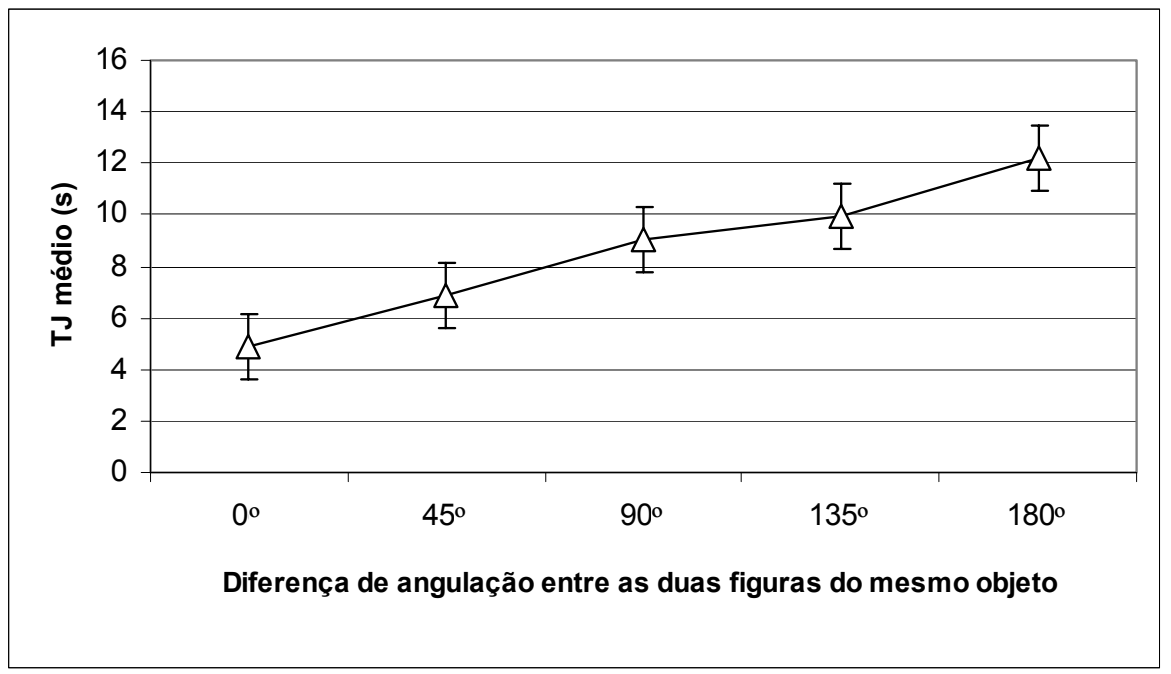

Figura 2 - Média do TJ nas comparações das figuras do objeto A em relação ao ângulo de rotação

A fim de compreender o padrão de busca visual e as estratégias na rotação mental, foram conduzidas análises pormenorizadas nos dados do objeto A. ANOVAS unifatoriais, em relação ao gênero, não mostraram diferença entre homens e mulheres para nenhuma das variáveis dependentes. No entanto, ANOVAS unifatoriais em relação à diferença de angulação entre as figuras do objeto A indicam efeitos significativos para todas as variáveis.

A Tabela 1 sumaria a média e desvio padrão das respostas corretas. Conforme esperado, a freqüência de respostas corretas diminuiu com o aumento do ângulo de rotação, $F(4,195)=2,739, p=0,030$. Também conforme esperado, foram observados aumentos lineares em virtude do grau de angulação para: tempo de julgamento, $F(4,163)=12,04, p<0,000$; número total de fixação nos objetos, $F(4,163)=10,202$, $p=0,000$; número de fixação no objeto do lado esquerdo, $F(4,163)=8,475, p=0,000$; número de fixação no objeto do lado direito, $F(4,163)=9,929$, $p=0,000$; duração total das fixações no objeto, $F(4,163)=12,373, p=0,000$; duração total das fixações no objeto do lado esquerdo, $F(4,163)=$ $10,325, p=0,000$; duração total das fixações no objeto do lado direito, $F(4,163)=11,313, p=0,000$ e número de alternâncias entre os dois objetos, $F$ (4, $163)=8,008, p=0,000$. O número de alternâncias aumentou em média uma vez a cada $45^{\circ}$.

Tabela 1 - Média e desvio padrão dos acertos, tempo de julgamento em segundos (TJ), número de fixações (NF), número de fixação esquerda (NFE), número de fixação direita (NFD), duração das fixações em segundos (DF), duração das fixações esquerda (DFE), duração das fixações direita (DFD) e número de alternâncias, das respostas corretas em função das diferenças de angulação

\begin{tabular}{lcccccccccc}
\hline \multirow{2}{*}{ Variáveis } & \multicolumn{3}{c}{$0^{\circ}$} & \multicolumn{2}{c}{$45^{\circ}$} & \multicolumn{2}{c}{$90^{\circ}$} & \multicolumn{2}{c}{$135^{\circ}$} & \multicolumn{2}{c}{$180^{\circ}$} \\
& Média & DP & Média & DP & Média & DP & Média & DP & Média & DP \\
\hline Acertos & 0,97 & 0,15 & 0,90 & 0,30 & 0,77 & 0,42 & 0,75 & 0,44 & 0,80 & 0,40 \\
TJ & 4,89 & 1,70 & 6,87 & 4,42 & 9,02 & 3,95 & 9,90 & 4,53 & 12,20 & 7,82 \\
NF & 16,51 & 5,76 & 21,30 & 11,92 & 27,19 & 11,61 & 29,23 & 13,01 & 35,16 & 21,13 \\
NFE & 7,61 & 2,91 & 10,30 & 7,06 & 13,16 & 6,21 & 13,17 & 6,10 & 16,47 & 10,26 \\
NFD & 8,90 & 3,20 & 11,00 & 5,30 & 14,03 & 6,17 & 16,07 & 7,42 & 18,69 & 12,16 \\
DF & 4,02 & 1,65 & 5,84 & 3,86 & 7,82 & 3,74 & 8,47 & 3,87 & 10,59 & 6,83 \\
DFE & 1,89 & 8,06 & 2,94 & 2,42 & 3,84 & 1,78 & 3,84 & 1,71 & 4,99 & 3,30 \\
DFD & 2,13 & 0,92 & 2,90 & 1,51 & 3,98 & 2,30 & 4,63 & 2,37 & 5,59 & 4,05 \\
Alternâncias & 7,02 & 2,77 & 8,89 & 4,49 & 11,03 & 5,81 & 12,30 & 6,31 & 13,59 & 7,59 \\
\hline
\end{tabular}

Psico-USF, v. 10, n. 1, p. 41-49, jan./jun. 2005 
Foram analisados os traçados de busca visual dos 168 julgamentos corretos e 32 incorretos das figuras do objeto A, a fim de identificar os padrões de busca visual. A inspeção visual de todos traçados revelou a existência de cinco padrões diferentes na realização da tarefa. Esses padrões foram identificados com base na distribuição e localização das fixações e alternâncias nos objetos.

O padrão 1 se caracteriza pela concentração das fixações em apenas um eixo de alternâncias na parte superior dos objetos. No padrão 2, ocorre concentração das fixações e alternâncias na parte superior e no máximo duas alternâncias na parte inferior do objeto. $\mathrm{O}$ padrão 3 é composto por dois eixos bem definidos, com as fixações e alternâncias concentradas na parte superior e inferior dos objetos. O padrão 3 se diferencia do 2 pelo fato de apresentar mais de duas alternâncias na parte inferior. No padrão 4, o participante faz uma varredura em alternância dos objetos de cima para baixo, ou seja, as fixações e alternâncias estão igualmente distribuídas ao longo do objeto. Por fim, no padrão 5 , há um grande número de fixações em cada objeto e pouca alternância de lado. A Figura 3 ilustra os cinco tipos de padrões identificados pela inspeção visual.

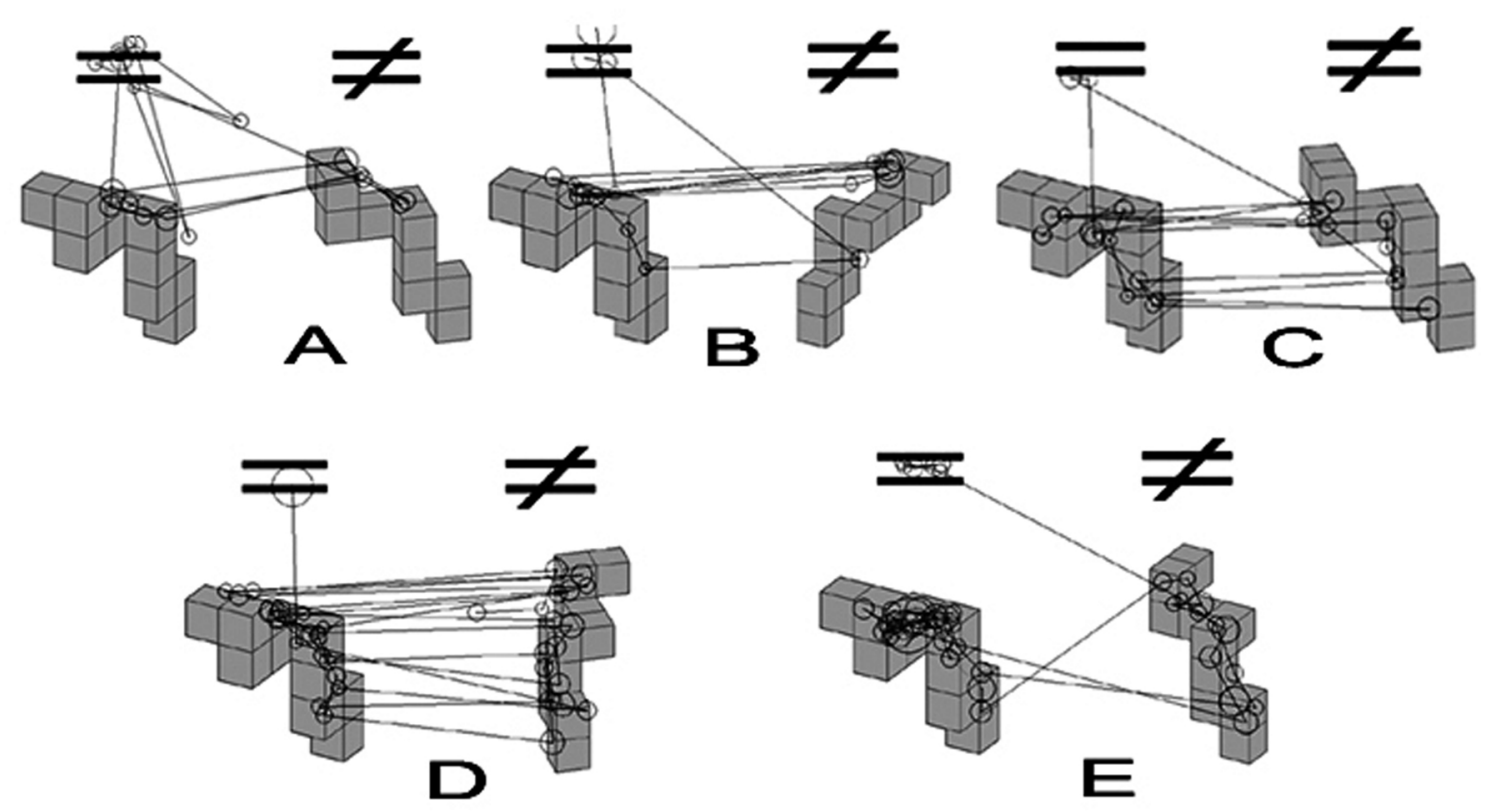

Figura 3 - Exemplos dos cinco tipos de padrões de busca visual, com os padrões 1, 2 e 3 na parte superior e os padrões 4 e 5 na inferior

A análise da freqüência de ocorrência dos cinco padrões foi feita por dois juízes, com índice de concordância de 83,5\%. As respostas discordantes foram analisadas por um terceiro juiz, que auxiliou na identificação dos padrões.

Foi observado o uso de mais de um padrão em $85 \%$ dos participantes. Em 42,5\% dos participantes (oito homens e nove mulheres), foram usados dois tipos de padrão, de acordo com a angulação, e 42,5\% (sete homens e 10 mulheres) chegaram a usar três. Quinze por cento (cinco homens e uma mulher) usaram apenas um padrão.

A Tabela 2 sumaria a distribuição do padrão de busca visual em relação à angulação para os julgamentos corretos (C) e incorretos (I). A freqüência de ocorrência dos padrões em ordem decrescente foi 4, 2, 3, 1 e 5 . No entanto, apenas os padrões 2,4 e 3 produziram freqüência de acertos significativamente maior que a de erros. 
Tabela 2 - Distribuição dos padrões de busca visual em relação à angulação para os julgamentos corretos (C) e incorretos (I)

\begin{tabular}{lcccccccccccccccc}
\hline & \multicolumn{3}{c}{ Padrão 1 } & \multicolumn{3}{c}{ Padrão 2 } & \multicolumn{3}{c}{ Padrão 3 } & \multicolumn{3}{c}{ Padrão 4 } & \multicolumn{3}{c}{ Padrão 5 } \\
\hline Angulação & C & I & T & C & I & T & C & I & T & C & I & T & C & I & T \\
\hline $0^{\circ}$ & 5 & 0 & 5 & 22 & 0 & 22 & 2 & 0 & 2 & 10 & 0 & 10 & 0 & 1 & 1 \\
$45^{\circ}$ & 2 & 1 & 3 & 16 & 1 & 17 & 5 & 0 & 5 & 13 & 1 & 14 & 0 & 1 & 1 \\
$90^{\circ}$ & 1 & 1 & 2 & 9 & 1 & 10 & 5 & 2 & 7 & 14 & 5 & 19 & 1 & 1 & 2 \\
$135^{\circ}$ & 0 & 2 & 2 & 10 & 3 & 13 & 3 & 0 & 3 & 16 & 4 & 20 & 1 & 1 & 2 \\
$180^{\circ}$ & 0 & 2 & 2 & 10 & 2 & 12 & 2 & 1 & 3 & 20 & 1 & 21 & 1 & 1 & 2 \\
\hline Total & 8 & 6 & 14 & 67 & 7 & 74 & 17 & 3 & 20 & 73 & 11 & 84 & 3 & 5 & 8 \\
\hline
\end{tabular}

Para avaliar a eficácia dos padrões, foi calculada a média do TJ para cada padrão. A Tabela 3 sumaria o TJ médio e o desvio padrão nas comparações corretas.
Os TJs médios descrevem uma função linear, sendo os padrões 1,2 e 3 com os menores tempos e os 4 e 5 com os maiores.

Tabela 3 - Tempo de julgamento médio em segundos em relação ao padrão

\begin{tabular}{cccccc}
\hline & Padrão 1 & Padrão 2 & Padrão 3 & Padrão 4 & Padrão 5 \\
\hline TJ médio & 4,40 & 6,05 & 6,10 & 10,72 & 19,96 \\
Desvio padrão & 1,09 & 2,76 & 2,54 & 6,32 & 8,04 \\
\hline
\end{tabular}

\section{Discussão}

Estudos mostram um efeito da angulação sobre o tempo de julgamento na rotação de objetos tridimensionais (Bauer \& Jolicoeur, 1996; Bryden, George \& Inch, 1990; Capovilla et al., 1998; Desrocher et al., 1995; Just \& Carpenter, 1976, 1985; Sanders et al., 1982; Shepard \& Metzler, 1971). No presente estudo esse efeito foi encontrado na rotação do objeto, evidenciando a hipótese de equivalência funcional (Sternberg, 2000).

A duração média das fixações, o número de fixações nas figuras e o número de alternâncias entre as duas figuras aumentaram como função direta do ângulo de rotação, confirmando os achados de Just e Carpenter (1976). A hipótese de que a rotação mental seja monitorada a cada $45^{\circ}$ é evidenciada por esses dados, pois a cada aumento de $45^{\circ}$ na angulação das figuras houve o acréscimo de uma alternância. Desta forma, a hipótese de rotação balística, na qual o participante faria uma rotação previamente determinada, é refutada. Se isso acontecesse, possivelmente, o tempo de julgamento e a duração das fixações sofreriam efeito da angulação, mas não haveria aumento significativo no número de alternâncias de lado, pois essas alternâncias ocorrem quando os participantes estão transformando e comparando um segmento da figura (Just \& Carpenter, 1976).

Com base na inspeção visual dos traçados da busca visual nessa tarefa, propõe-se que, para realizar a transformação, o indivíduo deva rotacionar um dos segmentos, mantê-lo na memória de trabalho (Baddeley \& Lieberman,1980) e compará-lo com o mesmo segmento do outro objeto. Deve repetir esse procedimento até que os objetos estejam idênticos. Uma rotação monitorada a cada $45^{\circ}$ significa que, quando a diferença for maior, o objeto mantido na memória de trabalho deverá sofrer mais uma rotação e ser comparado novamente. E, assim, sucessivamente, até os $180^{\circ}$ de diferença.

Convém lembrar que, segundo Just e Carpenter (1976), o processo não é composto somente pela transformação do segmento, mas pela busca de um segmento a ser rotacionado e pela confirmação. Tal fato explica por que é encontrada muito mais que apenas uma alternância na comparação de objetos rotacionados a $45^{\circ}$. Este estudo não se propunha a identificar os três estágios, mas apenas analisar a existência de padrões de busca visual em figuras rotacionadas no eixo $\mathrm{y}$.

A análise dos padrões de busca visual indica o uso de dois tipos de estratégias na realização da tarefa: rotação mental em torno dos eixos padrões e comparação independente da orientação (Just \& Carpenter, 1985). A rotação mental em torno dos eixos padrões foi encontrada nos padrões 1, 2, 3 e 4. Nesses padrões, as alternâncias das fixações entre os objetos do lado esquerdo e direito sugerem que os participantes tenham rotacionado mentalmente segmentos do objeto no eixo y. Essa estratégia foi encontrada em 97,5\% dos participantes, corroborando as discussões de Shepard e Metzler (1971) segundo as quais a rotação mental é a estratégia mais usada para resolver esse tipo de problema.

A comparação independente da orientação foi encontrada em apenas um participante, quando o julgamento foi correto, e foi representada pelo padrão 5. Nesse padrão de busca visual há um grande número de fixações em cada objeto e pouca alternância entre eles. 
Isso sugere que o participante não tenha feito rotação mental, mas sim análise pormenorizada de cada objeto a fim de comparar os objetos quanto a suas características espaciais. Tais dados corroboram a proposição de Just e Carpenter (1985), segundo a qual essas duas estratégias podem ser encontradas nas tarefas de Shepard e Metzler com supremacia do uso da rotação mental.

Dessa forma, os diferentes padrões de busca visual não necessariamente implicam estratégias distintas. As diferenças encontradas entre os padrões 1, 2, 3 e 4 se baseiam na distribuição e localização das fixações e alternâncias entre os objetos. Nota-se a formação de eixos de comparação nos padrões 1, 2 e 3, com maior concentração localizada na parte superior dos objetos.

Apenas 20\% dos participantes usaram o mesmo padrão de busca para todas as angulações. O uso de diferentes padrões por um mesmo participante pode ser explicado de duas maneiras. A primeira seria o estabelecimento do learning set (Catania, 1999), em que a adoção de um mesmo padrão se daria somente baseada em um número específico de treinos. A segunda seria a influência da angulação dos objetos no uso dos padrões. Um estudo comparando os padrões de busca visual de indivíduos que passassem por um treino na tarefa com os de participantes não-treinados seria importante para testar essas hipóteses.

O padrão 1 teve baixa freqüência e foi encontrado somente nos julgamentos a $0^{\circ}, 45^{\circ}$ e $90^{\circ}$. A concentração das fixações e alternâncias em apenas um eixo sugere que a transformação realizada não necessita de confirmação, pois as figuras têm pouca ou nenhuma diferença de angulação. O padrão 2 foi encontrado em todas as angulações, mas se concentrou em $0^{\circ}$ e $45^{\circ}$. Nesse tipo de busca o participante transforma o segmento superior e faz poucas fixações para confirmar a rotação nos segmentos inferiores. Ele possivelmente não realiza outra transformação, mas apenas verifica se as mesmas relações espaciais entre os segmentos inferiores são mantidas em ambos os objetos.

No padrão 3, a concentração das fixações em dois eixos indica que a confirmação nos segmentos inferiores tenha sido feita por meio de outra transformação. Esse padrão teve baixa incidência e distribuição homogênea entre as angulações. E, por fim, no padrão 4 o participante faz uma varredura, como se estivesse transformando e confirmando a rotação em cada segmento dos objetos. Este padrão é encontrado em todas as angulações e é mais freqüente a $90^{\circ}, 135^{\circ}$ e $180^{\circ}$.

Dessa forma, a distribuição do uso dos padrões em relação ao ângulo de rotação sugere que um aumento na diferença de angulação acarreta no uso de padrões mais complexos, sendo o padrão 1 mais simples e o 4, mais complexo.

O desempenho na tarefa pode ser avaliado de acordo com o tempo de julgamento correto. Tempos menores indicam bom desempenho e tempos maiores, mau desempenho. Nota-se que os padrões 1, 2 e 3 consumiram menos tempo, e os padrões 4 e 5 , mais tempo. Isso pode ser explicado em virtude de os primeiros terem sido mais usados entre as rotações de $0^{\circ}$ a $90^{\circ}$ que, em geral, levam menos tempo para serem julgadas.

A comparação entre a freqüência de ocorrência do padrão e do tempo médio consumido pode ser considerada fator indicativo de eficiência. Considerando-se a freqüência de ocorrência, os padrões 2 e 4 foram os mais usados, produzindo alta taxa de acertos. No entanto, o tempo consumido no padrão 4 foi maior que no 2. Portanto, o padrão 2 pode ser considerado o mais eficiente de todos.

É possível que o desempenho nas tarefas de rotação mental esteja associado à maior capacidade de manter a informação na memória de trabalho visual, a tela de esboço visuoespacial (Baddeley \& Lieberman, 1980). Assim, independentemente do padrão de busca visual adotado, seriam necessárias menos fixações e alternâncias e, portanto, menos tempo. A relação entre a estratégia de comparação independente da orientação (padrão 5) e o mau desempenho foi significativa. Nesse caso, a necessidade de manter na memória de trabalho maior número de informações para comparar os objetos desencadeou aumento no tempo de julgamento e duração das fixações.

Não foram encontradas diferenças entre homens e mulheres na realização da tarefa. É possível que a rotação em apenas um eixo tenha facilitado a tarefa, diminuindo a diferença entre homens e mulheres citada na literatura (Bryden et al., 1990; Capovilla et al., 1998; Covre, Piza, Lukasova \& Macedo, 2002; Desroscher et al., 1995; Kimura, 1999; Sanders et al., 1982). Os padrões de busca visual não sofreram efeito de gênero, mas os padrões e as estratégias menos usados foram feitos por homens.

\section{Referências}

Baddeley, A. D. \& Lieberman, K. (1980). Spatial working memory. Em R. S. Nickerson (Ed.). Attention \& performance (vol. VIII). Hillsdale, NJ: Lawrence Erlbaum.

Bauer, B. \& Jolicoeur, P. (1996). Stimulus dimensionality effects in mental rotation. Journal of Experimental Psychology: Human Perception and Performance, 22(1), 82-94.

Bryden, M. P., George, J. \& Inch, R. (1990). Sex differences and the role of figural complexity in determining the rate of mental rotation. Perceptual and Motor Skills, 70, 467-477. 
Capovilla, F. C., Guedes, M. \& Macedo, E. C. (1998). Análise computadorizada de rotação mental de objetos tridimensionais: efeitos de diferença de angulação e de eixo de rotação II. Ciência Cognitiva Teoria, Pesquisa e Aplicação, 2(4), 721-820.

Catania, A. C. (1999). Aprendizagem: comportamento, linguagem e cognição. Porto Alegre: Artes Médicas.

Covre, P., Piza, C. M. J. T., Lukasova, K. \& Macedo, E. C. (2002). Diferenças de gênero na capacidade de rotação mental de objetos. Boletim de Iniciação Cientifica em Psicologia da Universidade Presbiteriana Mackenzie, 3(1), 19-26.

Desrocher, M., Smith, M. L. \& Taylor, M. (1995). Stimulus and sex differences in performance of mental rotation: Evidence form event related potentials. Brain and Cognition, 28, 14-38.

Fischer, M. H., Deubel, H., Wohlschlager, A. \& Schneider, W. (1999). Visuomotor mental rotation of saccade direction. Experimental Brain Research, 127, 224-232.

Ganis, G., Keenan, J. P., Kosslyn, S. M. \& PascualLeone, A. (2000). Transcranial magnetic stimulation of primary cortex affects mental rotation. Cerebral Cortex, 10(2), 175-180.

Just, M. A. \& Carpenter, P. A. (1976). Eye fixations and cognitive process. Cognitive Psychology, 8, 441-480.

Just, M. A. \& Carpenter, P. A. (1985). Cognitive coordinate systems: Accounts of mental rotation and individual differences in spatial ability. Psychological Review, 92(2), 137-172.

Kimura, D. (1999). Sex and cognition. Boston, MA: A Bradford Book.

Rayner, K. (1998). Eye movements in reading and information processing: 20 years of research. Psychological Bulletin, 124(3), 337-422.

Sanders, B., Soares, M. P. \& D’Aquila, J. M. (1982). The sex difference on one test of spatial visualization: A nontrivial difference. Child Development, 53, 1106-1110.

Shepard, R. \& Metzler, J. (1971). Mental rotation of three-dimensional objects. Science, 171(3972), 701-703.

Sternberg, R. J. (2000). Psicologia cognitiva. Porto Alegre: Artes Médicas Sul.

Wexler, M., Kosslyn, S. M. \& Berthoz, A. (1998). Motor processes in mental rotation. Cognition, 68, 77-94.

Wohlschlager, A. \& Wohlschlager, A. (1998). Mental and manual rotation. Journal of Experimental Psychology: Human Perception and Performance, 24, 397-412.

Wraga, M., Thompson, W. L., Alpert, N. M. \& Kosslyn, S. M. (2003). Implicit transfer of motor strategies in mental rotation. Brain and Cognition, 52, 135-143.

Recebido em julho de 2004 Reformulado em novembro de 2004 Aprovado em junho de 2005

Sobre os autores:

Priscila Covre é psicóloga, foi bolsista da Universidade Presbiteriana Mackenzie, e atualmente é mestranda em Psicobiologia na Universidade Federal de São Paulo.

Elizeu Coutinho de Macedo é psicólogo, mestre e doutor em Psicologia Experimental pela Universidade de São Paulo; e atua como professor de graduação e pós-graduação na Universidade Presbiteriana Mackenzie.

Fernando César Capovilla é psicólogo, mestre em Psicologia pela Universidade de Brasília, doutor em Psicologia Experimental pela Temple University of Philadelphia, livre-docente em Neuropsicologia Clínica pela Universidade de São Paulo e professor associado do Instituto de Psicologia da Universidade de São Paulo.

José Salomão Schwartzman é médico e doutor em Neurologia pela Universidade Federal de São Paulo, professor titular na Pós-Graduação em Distúrbios do Desenvolvimento da Universidade Presbiteriana Mackenzie e editor científico do periódico Temas sobre Desenvolvimento. 